\title{
Investigative Analysis and Comparison of WiMAX under Different Scenarios
}

\author{
Subarna Das \\ Chitkara University Institute of Engineering and \\ Technology \\ Chitkara University, Punjab, India
}

\author{
Shalli Rani \\ Chitkara University Institute of Engineering and \\ Technology \\ Chitkara University, Punjab, India
}

\begin{abstract}
Starting from a straightforward telephony application to various multimedia applications, must require a specific set of protocols. WiMAX is a concept of having different protocol sets that can be handpicked for an efficient transcribing process for data collection. BWA-MAN (Broadband Wireless Access to a Metropolitan Area Network) technology, based on the IEEE- based 802.16 standards, provides wireless-based access over vast distances in different techniques from a single point to multipoint point communication or multipoint to mobile telephony access. Lower cost deployment with a wider coverage area is one of the features of it. In the presented article, the simulation results of basic performance parameters of the WiMAX network like Data Rate, Throughput, Transmitted, and Received Packet Rate are studied, and the functionality of WiMAX is elaborated in detail. To investigate the features and functionality of the WiMAX network, a different scenario for the Static and Dynamic WiMAX network is developed. OPNET 14.0 was utilized in this research to investigate various performance statistics and to promote the different scenarios of WiMAX. The static WiMAX network is observed to be better as compared to the dynamic in some parameters, but the flexibility of mobility is to be compromised. The results obtained in work show the efficiency of the network for different requirements, present, and future demand. Further on, It is also investigated that Mobile WiMAX can be preferred for its features like sufficient internet speed and VoIP services.
\end{abstract}

\section{Keywords}

WiMAX, OPNET 14.0, Static and Dynamic WiMAX network, VoIP.

\section{INTRODUCTION}

Since its inception in the early 1990s, the enhancement of wireless technology in different areas of communicationbased environments and applications grow rapidly [7] [18]. In earlier stages since no specific global protocols were introduced for the effective communication and transmission of data between two or more points, the implementation is not sufficient, which tends to the introduction of two different universal protocols based standard system in 1999[17]. These are the IEEE 802.16d standard, which supports the fixed type wireless broadband access system with the only physical layer, and the MAC layer of the remote medium is introduced and IEEE 802.16e, which supports both wired as well as remote wireless systems[13][19]. WiMAX represents IEEE wireless MAN with its spectrum ranges from microwave to millimeterwave. The main functioning of WiMAX is to provide accessibility from one node to multiple nodes in the Metropolitan Area Network (MAN)[6][9]. A new set of protocols were introduced in 2003 i.e., IEEE 802.16a which is extended version of IEEE 802.16, which was effective only in open and clear areas having less penetration in the higher range and therefore, for the effective communication and data exchange where LOS (Line of Sight) communication is not possible due to extensive rise of buildings and structures [12]. IEEE 802.16a having Mesh topology preloaded with better QoS (Quality of Service), was preferred. Mobile-based video, voice messages, and Hotspot node generators with guaranteed QoS from the MAC layer were the reported features of then IEEE802.16a. Comparative features are discussed below in Table 1 between different IEEE standards [16]. WiMAX Forum [16] depicts WiMAX as "a standard based innovation empowering the conveyance of last-mile remote broadband access as a choice to link and digital subscriber line (DSL)." The primary purpose of developing the IEEE 802.16e \& the IEEE 802.20 standard because both are the new way of advanced wireless broadband by a new mobility system. In which both standards look the same, but there will be small important variances between them. The IEEE 802.16e is the one which will add portability in an authorized band, which is 2 to $6 \mathrm{GHz}$, but on another side, IEEE 802.20 keeps the main motive on operations in an authorized band which is under 3.5GHZ. By this, also can say that the dependent items which are based on IEEE 802.16e will be going to take the success of full arcade, and also, it will happen before 802.20 could make any solutions. The IEEE $802.16 \mathrm{e}$ standard is expanding the utilization of broadband remote access (BWA) by developing the "immutable portability of remote media." The alteration to 802.16 , which is additionally called the wireless metropolitan territory, organizes (WMAN) standard. Broadband Wireless Access (BWA) frameworks, for example, IEEE 802.16 norm, give fixed-remote access between the endorser station (private or business clients) and the Internet Specialist Organization (ISO) through the base station. BWA frameworks supplement existing last-mile wired systems, for example, link modem and DSL. Because of the up and coming air interface advancements, which guarantee to convey high transmission information rates, BWA frameworks become an appealing other option.

Table 1: Comparison of IEEE 802.16, IEEE 802.16a and IEEE 802.16e [16]

\begin{tabular}{|c|c|c|c|c|}
\hline Sr.no & Parameters & $\mathbf{8 0 2 . 1 6}$ & $\mathbf{8 0 2 . 1 6 a}$ & $\mathbf{0 2 . 1 6 e}$ \\
\hline $\mathbf{1}$ & Spectrum & $10-66$ & $2-11 \mathrm{GHz}$ & $2-6 \mathrm{GHz}$ \\
& & $\mathrm{GHz}$ & & \\
\hline $\mathbf{2}$ & Channel & $20,25 \mathrm{and}$ & 1.5 to 20 & 1.5 to 20 \\
& bandwidth & $28 \mathrm{MHz}$ & $\mathrm{MHz}$ & $\begin{array}{c}\mathrm{MHz} \\
\text { with UL } \\
\text { sub }\end{array}$ \\
& & & & \\
& & & \\
\end{tabular}




\begin{tabular}{|c|c|c|c|c|}
\hline & & & & channels \\
\hline 3 & Modulation & $\begin{array}{l}\text { QPSK.16 } \\
\text { QAM }\end{array}$ & $\begin{array}{c}\text { OFDMA } \\
256 \text { sub } \\
\text { Carriers } \\
\text { QPSK, } \\
16 \text { QAM, } \\
64 \text { QAM }\end{array}$ & $\begin{array}{c}\text { OFDMA } \\
256 \text { sub } \\
\text { carriers } \\
\text { QPSK, } 16 \\
\text { QAM, } \\
\text { 64 QAM }\end{array}$ \\
\hline 4 & Bit Rate & $\begin{array}{c}32 * 134 \\
\text { Mbps (28 } \\
\text { MHz) }\end{array}$ & $\begin{array}{c}75 \\
\text { Mb]bps } \\
\text { (20 MHz) } \\
\text { Non-LOS }\end{array}$ & $\begin{array}{l}15 \mathrm{Mbps} \\
(5 \mathrm{Mhz})\end{array}$ \\
\hline 5 & $\begin{array}{l}\text { Channel } \\
\text { conditions }\end{array}$ & LOS & Non-LOS & Non-LOS \\
\hline 6 & $\begin{array}{l}\text { Typical cell } \\
\text { radius }\end{array}$ & $2-5 \mathrm{Km}$ & $\begin{array}{c}\text { 7-10 Km, } \\
\text { Max } \\
50 \mathrm{Km}\end{array}$ & $2-5 \mathrm{Km}$ \\
\hline 7 & Application & Fixed & $\begin{array}{c}\text { Fixed and } \\
\text { portal }\end{array}$ & Mobility \\
\hline
\end{tabular}

\section{QOS STRUCTURE OF IEEE 802.16 STANDARD:}

WiMAX has four layers: MAC privacy, MAC sub-layer, Media Access convergence (MAC), and the last one is the physical layer. WiMAX MAC associated with the basis of connection like a connection should be recognized between a BS and SS before there will be a transmission could generate and on that way an association can be started either by a SS or by a BS and also the MAC sublayer is having the authority of providing the quality of service in WiMAX network. Every connection is distinguished by a one of a kind 16-bit connection identifier (CID), which is used to supervise various service flows. Service flow can be characterized as an arrangement of packets in single direction bearing, which are characterized by the same QoS parameters; for example, it is a unidirectional progression of parcels given a specific QoS. Each assistance stream is distinguished by an interesting 32bit identifier, known as a service flow identifier (SFID). In every service flow that can accompany one of the states: Dynamic, Conceded, and Provisioned, every state has an adequate related arrangement of QoS parameters. All the parameters are providing measurable service approximation, which can be called Delay, jitter, packet loss, etc. In the BS, the call admission control, which is also can be called CAC, and this is a module which is to analyze the request from different QoS parameters and defined that the application can be made successfully or not. IEEE 802.16 standard SS can encourage the various communicating services, e.g., video, audio, and data, with different QoS conditions. The MAC layer also characterized the QoS signaling mechanism, which controls the BS and SS data transmissions. From base station to subscriber station, the downlinking communication is quite simple, i.e., Because, through the downlink sub-frame, an only base station can transmit it. For uplink sub-frame transmission of a subscriber station in which time slots are provided by only base station $\&$ at the starting of each frame, the base station UL-MAP spread the information. From subscriber station to base station uses TDMA media access
control(MAC), and this is one of the approaches for uplink intercession.

In summary IEEE 802.16 defines three schedulers are united in this norm: Base station(BS) UL scheduler (chooses that on a particular time which subscriber station will transfer the data \& also \& UL for UGS service flow), Base-station DL scheduler (which controls the broadcasting between basestation to subscriber station for example in the DL route). \& the subscriber station (SS) scheduler (SS scheduler is liable for circulating data transfer capacity, which is designated to the SS by the uplink scheduler, among its dynamic connections). The IEEE 802.16 standard will not explain the UL scheduling for rtPS, nrtPS, BE service flow \& the controlling system of admission.

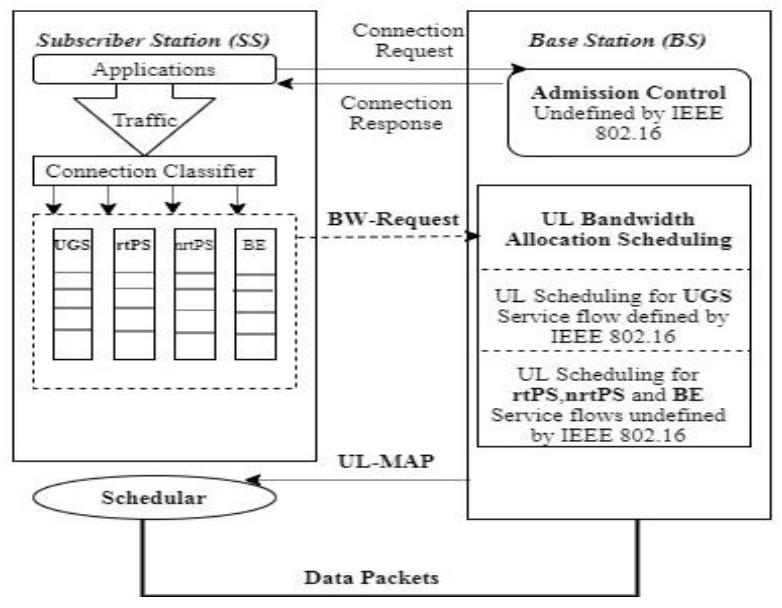

Fig.1: Architecture of IEEE 802.16 QoS.

In this fig.3 has shown the different QoS architecture for some other type of standards. For supporting various kinds of applications, the QoS classes provide five types of standards which are: - (A) UGS: It defines as Unsolicited grant service, which is mainly proposed for services like constant bit rate (CBR), e.g., VOIP. It is to complete the low Jitter \& low latency and, at the same time, the possibility of fewer packet drops. (B) rtPS: - The real-time polling service is intended to support some real-time service flows that are used to produce some variable sizes of data packets periodically like video streaming(MPEG). (C) ertPS: - The Extended real-time polling service is based on the effectiveness of UGS and as well as rtPS. It is intended to help UGS like service flows, which can get idle for an intermission like VoIP with silence destruction. (D) nrtPS: - Non- real-time polling service supports the flows which require the variable sizes of data packets and a less data rate feature like FTP. (E) BE:- Best effort service is intended for some applications that not required any less approved rate, which can be handle based on the best available sources \& it also not needed any QoS. In table 1 defines the different QoS parameters related to each class.

Table 2: Different QoS parameters related to its class

\begin{tabular}{|c|c|c|}
\hline Service & Description & QOS parameters \\
\hline UGS & $\begin{array}{c}\text { Designed to support real } \\
\text { time constant bit rate } \\
\text { (CBR) traffic such as VoIP } \\
\text { that periodically generates } \\
\text { fixed size data packets. }\end{array}$ & $\begin{array}{c}\text { Maximum } \\
\text { sustained rate, } \\
\text { maximum latency } \\
\text { tolerance }\end{array}$ \\
\hline RTPS & Designed to support MPEG & Maximum \\
\hline
\end{tabular}




\begin{tabular}{|c|c|c|}
\hline & $\begin{array}{l}\text { video \& teleconferencing } \\
\text { that periodically generates } \\
\text { variable size data packets. }\end{array}$ & $\begin{array}{c}\text { reserved rate, } \\
\text { maximum } \\
\text { sustained rate, } \\
\text { maximum latency } \\
\text { tolerance, traffic } \\
\text { priority. }\end{array}$ \\
\hline NRTPS & $\begin{array}{l}\text { Designed to support non } \\
\text { real time application with } \\
\text { minimum rate such as ftp. }\end{array}$ & $\begin{array}{l}\text { Minimum reserved } \\
\text { rate, maximum } \\
\text { sustained rate, } \\
\text { traffic priority. }\end{array}$ \\
\hline $\mathrm{BE}$ & $\begin{array}{l}\text { Designed to support data } \\
\text { stream which do not } \\
\text { require minimum service } \\
\text { level guarantee. This QOS } \\
\text { is used for internet service } \\
\text { such as email and web } \\
\text { browsing. }\end{array}$ & $\begin{array}{l}\text { Maximum } \\
\text { sustained rate, } \\
\text { traffic priority. }\end{array}$ \\
\hline ERTPS & $\begin{array}{l}\text { Designed to support real } \\
\text { time application, such as } \\
\text { VoIP with silence } \\
\text { suppression that have } \\
\text { variable data rate. }\end{array}$ & $\begin{array}{l}\text { Minimum reserved } \\
\text { rate, maximum } \\
\text { sustained rate, } \\
\text { maximum latency } \\
\text { tolerance }\end{array}$ \\
\hline
\end{tabular}

\section{RELATED WORK}

In [1], the number of VoIP calls that can be stabilized by the network available with adequate quality of services and having the provision of further addition or extension in the capacity for the present future network. Further, they explain the selection and settings of the underlying protocol for simulation configurations. Specific issues like quality of services, the flow of data, and the impact of traffic acting in the background. In [2], the problems occurred during the communication of video packets in video- based calls, during video meetings and other multimedia applications and services on the WiMAX. Point to Multipoint topology is defined and modeled. In this paper, the author explains the performance analysis as a change in traffic flow like Throughput, packet loss, and average delay. In [3], RTPS when extends the arranging facility and upkeep VRRT, which further improves the performance of different applications like VoIP over WiMAX.

In [4], the voice quality level with satisfying jitter values and good latency with four on the MOS scale elaborated also explains the bandwidth effect on the jitter in the networks. In [5], various Radio Access Technologies (RATs) are coming together, which provides effective communication between the sources for different uses or applications like multimediabased streaming and IP traffic over voice. With the increase in demand because of traffic increase, these networks increase rapidly. To satisfy the demand, which is to provide effective quality of WiMAX components at all the locations at any time. So for that, a suitable model is designed. A simulation study was conducted that elaborate performance of WiMAX. a simulation study has been conducted to examine the performance of different quality based parameters of Universal Mobile Telecommunication Services and WiMAX for supporting VoIP. Simulation Modules are designed in Optical Networks (OPNET), and after extensive simulations end to end delay, jitter and PDF were evaluated, and during the evaluation, it was WiMAX which performs better than Universal Mobile Telecommunication Services. In [6], the GSM-EFR \& GSM-FR achieve required desirable quality of speech with very less delay and instant jitter when compared with VoIP. In [7] has compared the presentation of Wi-Fi and WiMAX situations and observed at their Throughput and load. WiMAX throughput is higher in the event of heavier traffic, and wide territory go. WiMAX may deal with heavier load contrasted with Wi-Fi. By reproduction results, it is demonstrated that the WiMAX lining delay is littler because WiMAX offers broadband support to convey traffic load over the system cumbersomely. Lining delays for both the Wi-Fi summary of a plot are indistinguishable. They considered different parameters, for example, deferral, burden, and Throughput of the base station, switch, and endorser station and broke down their impact on the exhibition of WiMAX in a neighborhood. The base station or those switch holds up in Wi-Fi were thought about, and, true to form, the delay in the Wi-Fi switch was higher than the deferral in the base station. WiMAX is progressively proficient for the demonstration of taking care of more information with less lining defer when contrasted with Wi-Fi. In [8] introduced their examination on different fundamental WiMAX QoS parameters, which are basic in deciding the presentation of a WiMAX arrange. Low estimation of postponement, bundle misfortune, and jitter is accomplished, while a high normal estimation of Throughput and parcel conveyance is acquired. Further, they have dissected the parameters, for example, MOS, parcel start to finish delay, normal in voice bundle, and voice jitter, wherein G.711 codec is the best as far as MOS, and G.723 codec is best as far as parcel start to finish delay. In conclusion, they have reasoned that the BE, rtPS, and UGS administration classes utilizing distinctive VoIP codecs have been reproduced and broke down as far as normal jitter, Throughput, and normal deferral. The rtPS administration class comes out to be better than BE administration class for normal jitter. Something else, all the administration classes over VoIP codec's getting looked at work productively with regards to under six hubs. In[9] has led a broad investigation to assess the exhibition of UMTS and WiMAX for their response on VoIP traffic.it is also examined some significant basic parameters, for example, MOS, start to finish postponement, jitter, and bundle defers variety. The results show the UMTS is dominated in performance by WiMAX. In [10] introduced the development or advancement in estimating the exhibition of IEEE 802.16. they have used the WiMAX Connection insights (for example, Throughput, load) to determine traffic mapped conduct to support streams. For delay-reasonable traffic that shows variety outside its related rate, they have used ertPS booking class, which has the advantage of giving back a portion of its saved data transmission, if there is no traffic to be served by this held transfer speed. For voice quality over different situations, the exhibition of the two information and voice is consistent and comparable for MOS at approx. 2.8 to 360 seconds. In [11] has estimated the exhibition of a few VoIP codecs over the WiMAX organize. The system execution measurements, for example, parcel start to finish delay, MOS, bundle defer variety, and jitter, have been utilized to gauge the presentation of VoIP codecs. It is appeared by the outcomes that the codec G.723 offers the best outcomes among all talked about Codecs in completely presented execution measurements; the most noteworthy MOS, the least postponement, and the highest Throughput. In [12], the unauthorized and unlicensed UWB with a range of $5 \mathrm{GHz}$ having less pricing range than other types of a carrier -transmission technologies, but these unlicensed spectrums are having issues for non-line of sight based applications or where there is a continues fading signal like in rural areas. It is also explained that Time division Multiple Accessing is a solution to control the delay for longdistance based channels of WiMAX or Wi-Fi based 
connectivity. In this paper, it is explained that by implementing Time Division Multiple Access (TDMA), the fading and delay can be controlled for long-distance communication solutions and rural links. This research elaborates about the impact of finding out optimal functioning point for a connection where maximum Throughput is offered while controlling the delays to a minimum level i.e. $5 \mathrm{~ms}$.

\section{SIMULATION \& RESULTS}

The complete Scenario will be generated in Optical Network (OPNET) version 14.0 and defined features and accessibility of WiMAX Network will be investigated in terms of Data Rate, Throughput, Received and Transmitted rate etc. Single input node to multiple output node trajectories will be defined. Various Quality of Service factors of WiMAX are simulated by designing a network in Optical Networks (OPNET, 14.0). Outcomes for different scenarios are explained below.

\begin{tabular}{|c|c|}
\hline Parameter & Value \\
\hline Simulator & OPNET \\
\hline Simulation Time & 60 minutes \\
\hline Traffic Type & FTP \\
\hline Scalability & $200 \times 200 \mathrm{~m}_{2}$ \\
\hline Model Family & WIMAX \\
\hline Addressing Mode & IPV4 \\
\hline PHY Type & OFDMA \\
\hline
\end{tabular}

\subsection{Dynamic WiMAX Network}

\subsubsection{Delay}

It is observed that Packet delay for Dynamic WiMAX system is approaches to peak i.e. up to $0.041 \mathrm{sec}$ within 7 minutes (in first 10 minutes) and after that results shows that delay average value ranges from 0.026 to $0.028 \mathrm{sec}$.

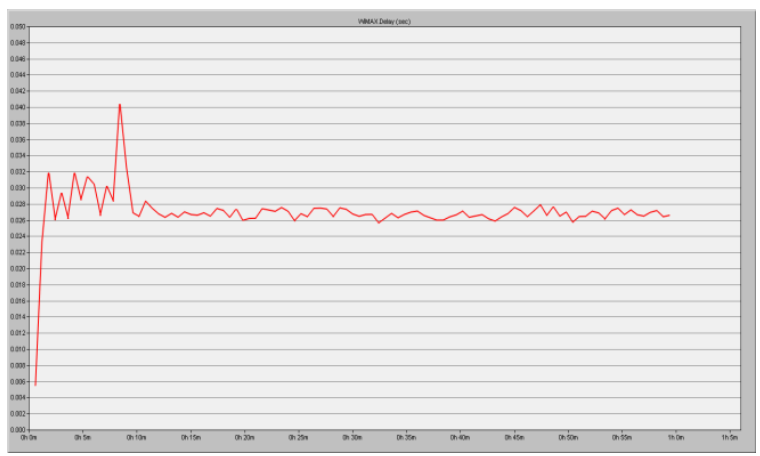

Figure 2: Variation in Delay in seconds for Wi-MAX Network

\subsubsection{Jitter}

Results for the Jitter parameter shows that the jitter average value in the WiMAX system ranges from 0.20 to $0.022 \mathrm{sec}$. It approaches to peak ie. $0.023 \mathrm{sec}$ in initial 10 minutes.

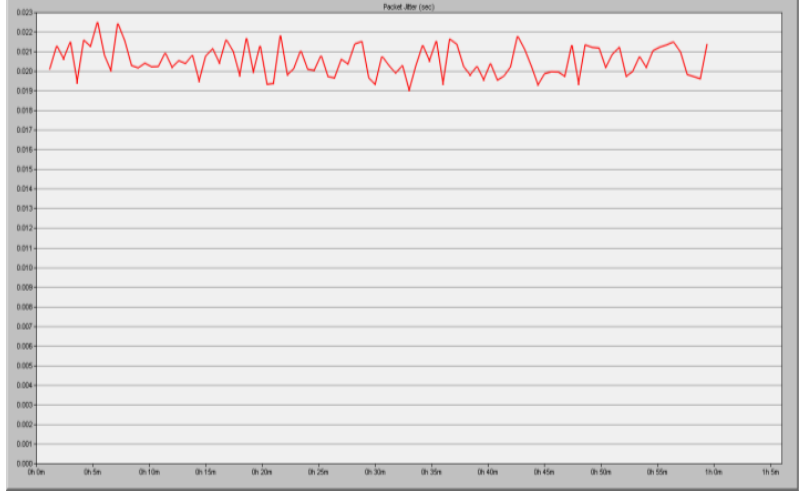

Figure 3: Variation in Jitter in seconds for Wi-MAX Network

\subsubsection{Throughput:}

Throughput can be calculated in bits/second. In figure 4 Results shows that initially the data transferred varies for first 7-10 mins and after that the transmission becomes constant. Once it attains maximum level in fraction of time after that it remains almost constant with very less up- down spikes throughout the scenario.

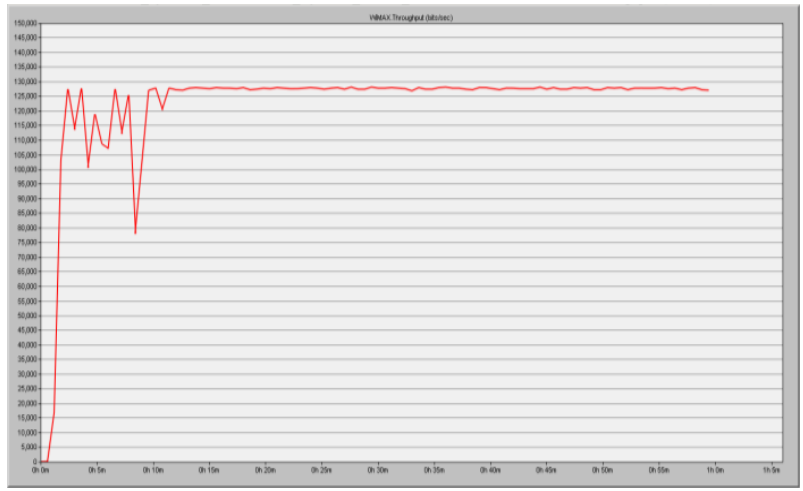

Figure 4: variation in Throughput in bits per second for Wi- MAX Network

\subsubsection{Traffic Sent:}

It is the data which is transmitted by the transmitters in a network in b/s. As shown in Figure 5, the transmission is very much constant.

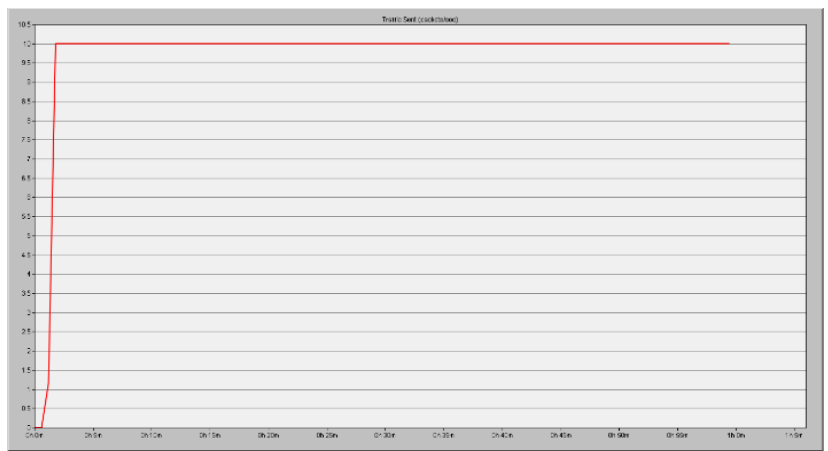

Figure 5: Traffic Transmission for Wi-MAX Network

4.1.5 Traffic Received:

It is the amount of data collected by the different receivers. Its units are in bits/sec. It is observed from the figure 6 about the amount of data which is received i.e. $64 \mathrm{~kb} / \mathrm{sec}$. 


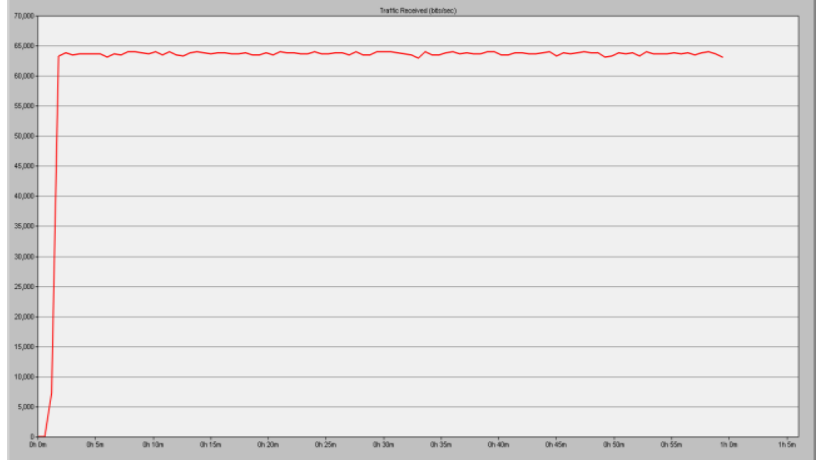

Figure 6: Traffic Acknowledged for Wi-MAX Network

\subsection{Static Wi-Max Network}

\subsubsection{Delay:}

It is observed that delay in the case of Static WiMAX network is almost constant or just deviates around 20-22 value as shown in graph.

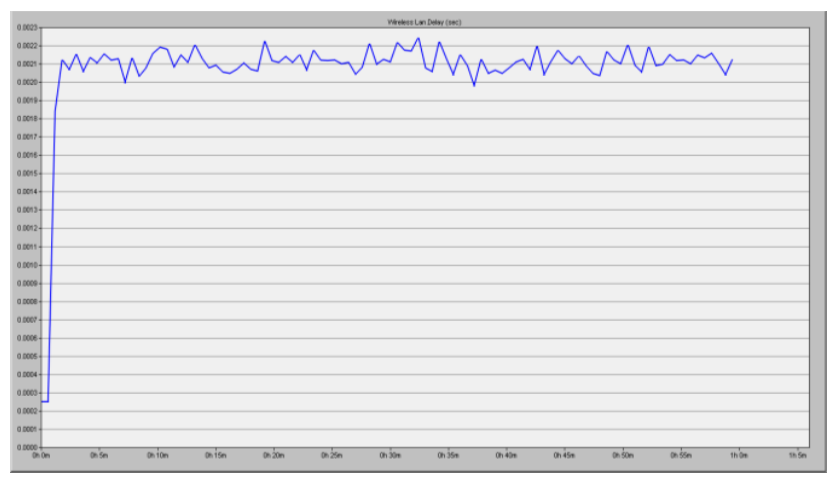

Figure 7: variation in Delay in seconds for Wi-MAX Network.

\subsubsection{Throughput:}

Throughput can be calculated in bits/second. In figure 8 Results explains that the transmission continuously deviates around a set of pattern as shown in graph and hence the performance declines when compare with Dynamic throughput.

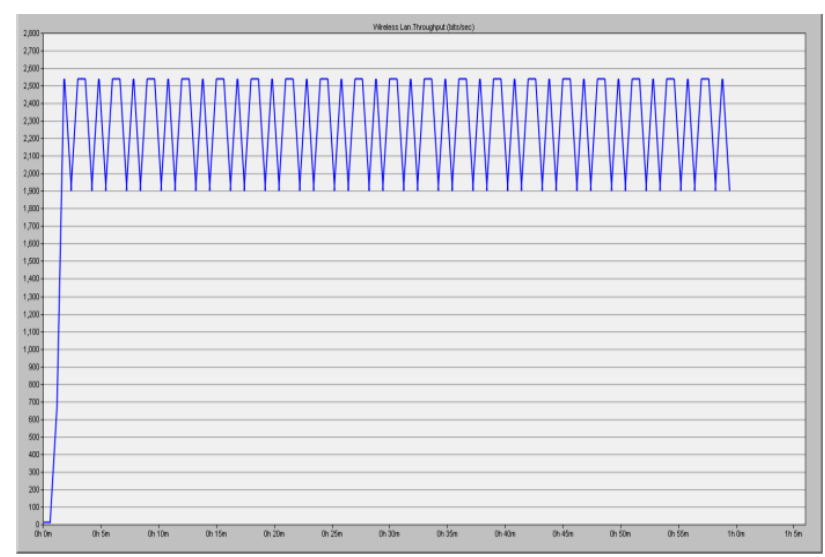

Figure 8: variation in Throughput in bits per second for WiMAX Network.

\subsubsection{Traffic Sent:}

It is the quantity of Packets sent during transmission process in bits/sec. The curve received is deviating curve which deviates in a constant pattern as shown in figure 9.

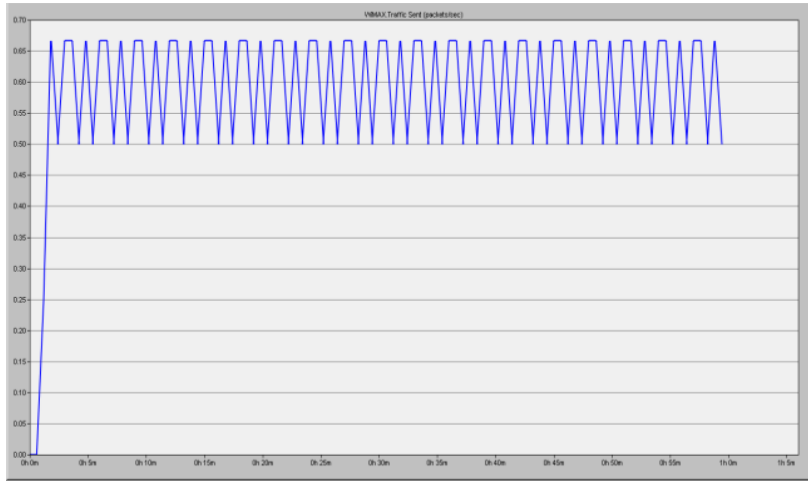

Figure 9: Traffic Sent for Wi-MAX Network

\subsubsection{Traffic Received:}

It is the quantum of Packets received in bits/sec.The curve received is deviating curve as compare to constant curve in dynamic WiMAX as it deviates in a constant pattern as shown in figure 9 .

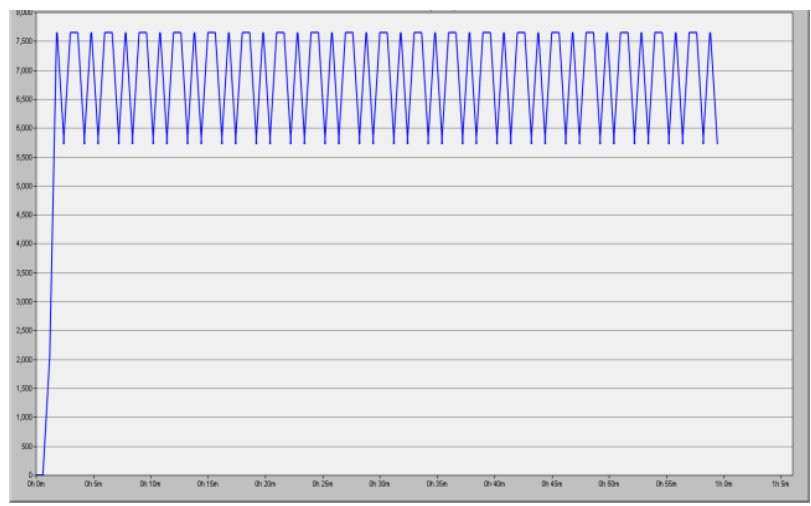

Figure10: Traffic Acknowledged Wi-MAX Network

\section{CONCLUSION}

In this Article IEEE standard based different Quality of Service attributes are investigated and compared on dynamic and static WiMAX scenarios, reflecting after simulations the performance of such networks, Outcomes explains that data delay occurred in the designed network exhibits very large in the starting phase as compare to during normal running of the simulation process. Jitter experiencing small change throughout the simulation process. For Throughput investigation which is an important factor indicates that the change is higher at initial stage and after then it remains constant. The results investigated in this research explains the performance comparison of both the networks for different requirements of present and future demand. It is also examined that dynamic WiMAX can be preferred for its features like effective internet speed and VoIP services.

\section{REFERENCES}

[1] K. Salah and A. Alkhoraidly, "An OPNET-based Simulation Approach for Deploying VoIP"in Journal International Journal of Network Management, Volume 16 Issue 3, Pages 159-183, May 2006.

[2] Nagarajan,N. and Rajeev, Kaarthick, "Performance Analysis of Video Conferencing and Multimedia Applications Services over WiMAX", International Advance Computing Conference(IAAC), 2009.

[3] Adi chandra, "Measuring data and Voip traffic in WiMAX networks," Journal of 
telecommunications,vol.2,no.1,pp.1- 6,2010.

[4] Rohani Bakar,Muhammad Ibrahim and D.M.Ali , "Performance Measurement of Voip over WiMAX 4g network", International colloquium on signal processing and its applications, 2012 .

[5] Jadhav , S., Zhang,H, \& Huang, "Performance Evaluation of Quality of Voip in WiMAX and UMTS", 12th International Conference on Parallel and Distributed Computing, Applications and Technologies, 2012.

[6] Tariq,M.I.,Azad,M.A.,Beuran,R. and shinoda,Y. "Performance analysis of voip codecs over BE WiMAX network," 3rd International conference on computer and electrical engineering(ICCEE 2010),vol .4,no.4,pp.7788,2012 .

[7] Nitish Meena and Nilesh Parihar, "Performance Existence Of Wifi And WiMAX Used Opnet", International Journal of Science,

[8] Technology \& Management, Volume No.03, Issue No. 12, 2014.

[9] Shibani Bagi, Anupama Sanjay, Awati Giridhar and S Sudi, "Analysis of VoIP Traffic in WiMAX Environment", International Journal of Modern Trends in Engineering and Research (IJMTER) Volume 02, Issue $02,2015$.

[10] Sheetal Jadhav, Haibo Zhang and Zhiyi Huang , "Performance Evaluation of Quality of VoIP in WiMAX and UMTS" in International Journal of Computer Applications (0975 - 8887) International Conference on Communication, Circuits and Systems "iC3S-2012" 2015.

[11] Neeru Mehta and leena,"Performance Analysis of VOIP over WiMAX", International Journal of Science, Engineering and Technology Research (IJSETR), Volume 4, Issue 6,2015.

[12] Sheetal Jalendry and Shradha Verma,"Performance Analysis of various Codecs Schemes of VOIP over WiMAX”, International Journal of Advanced Research in Computer Engineering \& Technology (IJARCET) Volume 4 Issue 6, 2015.
[13] JoaoHenriques, VitorBernado,PauloSimoes,MariliaCurad o, "Voip Performance over Mobile WiMAX: An Urban

[14] Deployment analysis", IEEE Communications, 2012.

[15] Chin-Ling Chen and Cheng-Yi Pan, "A Downlinkk Scheduling based on queue length estimation for Voip inn WiMAX Networks", 5thInternational Conference on Biomedical Engineering and Information (BMEI), 2012.

[16] Joao Henriques, Vitor Bernado, Paulo Simoes and Marilia Curado, "Voip Performance over Mobile WiMAX: An Urban Deployment analysis", IEEE Communications, 2012.

[17] Tarik Anouari and Abdel krim Haqiq, "Performance Analysis of VoIP Traffic in WiMAX using various Service Classes", International Journal of Computer Applications (0975 - 8887) Volume 52- No.20, 2012

[18] Navjot Kaur and Deepinder Singh , “ A review of an Adaptive On-Demand Routing Protocols for Mobile Adhoc Networks", International Journal of Computer Applications, proceedings of ICAET-2015, BGIET, 2015.

[19] Hiba Osman Mohammed Osman, Dr. Khalid Hamid Bilal and Dr. Amin Babiker Mustafa, "End to End Delay of VoIP over WiMAX", International Journal of Engineering, Applied and Management Sciences Paradigms, Vol. 35, Issue 01, 2016.

[20] Mishra, Chandrakant and Lal, Sonu,"Survey Paper on Performance Evaluation of WiMAX (IEEE 802.16) System using Space Time Block Coding Technique", JARCSSE, 6(1), 682-686,2016.

[21] Imam,Md. and Kumar, Amod, "Survey Paper on Perfromance Evaluation of 5G WiMAX (IEEE 802.16) System using Space Time Block Coding Technique", IRJET, Vol. 5, no. 8, pp. 1259-1262, 2018

[22] Javier Simo-Reigadas, Carlos Figuera, Eduardo Morgado, Esteban Municio and Andres MartınezFernandez, "Assessing IEEE 802.11 and IEEE 802.16 as Backhauling Technologies for 3G Small Cells in Rural Areas of Developing Countries", Hindawi Journal of Mobile Information Systems, Volume 2019, pp 1-16, 2019 